\title{
Fluorescent, Online Monitoring of PLGA Degradation for Regenerative Medicine Applications
}

\begin{abstract}
K. Bardsley, ${ }^{\mathrm{a}}$ I. Wimpenny, ${ }^{\mathrm{a}}$ Y. Yang, ${ }^{\mathrm{a}}$ and A. J. El Haj ${ }^{\mathrm{a}}$
Degradable polymers such as poly-lactic-co-glycolic acid (PLGA) are frequently chosen for tissue engineering, due to their ease of production, controllable degradation rates and Food and Drug Administration (FDA) approval. Within tissue engineering it is essential that the degradation profile of such biomaterials is understood and measured both in vitro and in vivo. The majority of techniques currently undertaken to study degradation are however destructive, leading to over reliance on end point analysis. This study therefore defines a method of fluorescently tagging PLGA, via the addition of reactive amine groups and subsequent isothiocyanate reactions, with the purpose of monitoring its degradation profiles through non-destructive techniques. The amine grafting and fluorescent labelling of the PLGA was confirmed using both x-ray photospectrometry and high performance liquid chromatography. The modification of the PLGA had no significant effect on molecular weight or the hydrophilicity of the polymer. Both the release of fluorescent biomaterials and the changes in fluorescent retention within the scaffold made from the fluorescently tagging PLGA was observed to be highly correlated to the changes in physical weight. This paper therefore demonstrates a novel method for the online and non-destructive monitoring of polymer degradation through the incorporation of a fluorescent marker, which can decrease the reliance on end point analysis and reduce the number of samples required both in vitro and in vivo.
\end{abstract}

\section{Introduction}

The field of tissue engineering often relies on the use of biomaterials as scaffolds to support the replacement of damaged tissue or guide natural repair. It is therefore essential that biomaterials are both biocompatible and biodegradable. Ideally biomaterial degradation should be at an equilibrium with the tissue formation and therefore allow for unimpeded new tissue formation, while retaining mechanical stability during repair. ${ }^{1,2}$ It is therefore essential that the degradation profile of biomaterials utilised within a tissue engineering therapy is well defined and does not hinder repair.

Current methods utilised for the degradation profiling of biomaterials include weight loss, high performance liquid chromatography (HPLC), gel permeation chromatography (GPC) and micro-computed tomography (microCT)..$^{3-6}$ These techniques are however destructive end-point analyses which do not allow for real-time monitoring of degradation. Recent advances in the area have utilised non-invasive, fluorescent techniques to tag biomaterials and assess degradation through subsequent changes in fluorescence. ${ }^{7}$ These fluorescent tagging approaches have been utilised on several biomaterials to date, including chitosan, ${ }^{8}$ PEG-dextran, ${ }^{7}$ collagen ${ }^{7}$ and fibrin, ${ }^{9}$ with correlations being observed between changes in fluorescence intensity and weight loss during degradation, both in vitro and in vivo. Non-destructive assessment of degradation decreases the reliance on end-point analysis and allows for continued sample monitoring both in vitro and in vivo. This is especially important in vivo when adhering the policies to replace, reduce and refine (3Rs) the use of animals in medical research, ${ }^{10}$ as when combined with online techniques for tissue formation or cell migration it allows for a decrease in the number of animals required for experimentation.

Synthetic, biodegradable polymers with simple chemistry and low reactivity groups are often used in tissue engineering to provide the required support for tissue regeneration as they can be easily and reproducibly manufactured. Poly(lactic-co-glycolic acid) is one such polymer which has been Food and Drug Agency (FDA) approved $^{11}$ and has been utilised in several tissue engineering applications including bone, cartilage, muscle and skin. ${ }^{12-15}$ One of the advantages of PLGA is that its degradation profile can be altered through the variation of the glycolic and lactic ratios and therefore it can be tailored to achieve maximum tissue regeneration. The relative inert reactivity of these polymers however means that there are no intrinsic molecular marks to detect or allow for the incorporation of active groups for degradation profile measurement. Hence degradation profiling of PLGA has relied heavily on destructive techniques.

In this paper we describe a novel method of fluorescently labelling PLGA using plasma treatment to produce an amine reactive polymer to which fluorescent molecule, rhodamine $b$ isothiocyanate, was bound. It was shown that the degradation profile of the fluorescent material obtained through changes in fluorescence was correlated to the loss in physical mass. This gives rise to a novel new method of tagging inert polymers with various amine reactive fluorochromes.

\section{Experimental}

Fluorescent PLGA fabrication 
PLGA with varying ratios of lactic to glycolic acid (50/50, 65/35 and 80/20) (Sigma-Aldrich, UK) were solvent cast in $20 \mathrm{~cm}$ glass dishes at a concentration of $2 \%(\mathrm{w} / \mathrm{v})$ in chloroform (fisher Scientific, UK) and left for 24 hours to allow for solvent evaporation. The PLGA films were then fluorescently labelled. Briefly the thin films (30 nm thickness) were plasma treated (Femto Low Pressure Plasma System, Diener Electronic, Germany) using concentrated ammonia (Sigma-Aldrich, UK) for 5 minutes under vacuum to add an amine group to the surface of the PLGA. The films were then reacted with rhodamine $b$ isothiocyanate $(50 \mu \mathrm{g} / \mathrm{mL}$ in $0.1 \mathrm{M}$ sodium carbonate (pH 9.0)) (Sigma-Aldrich, UK) at room temperature overnight in the dark on a tube roller [8]. PLGA was then washed with alternating washes of $70 \%(\mathrm{v} / \mathrm{v})$ ethanol (Fisher Scientific, UK) and distilled water to remove residual unreacted rhodamine $b$ isothiocyanate, until negligible fluorescence levels were observed within the wash solutions. PLGA films were lypholised, overnight at $-50{ }^{\circ} \mathrm{C}$ under a vacuum, and either sent for analysis or re-cast into three-dimensional (3D) scaffolds through the process of salt leaching. This process of recasting ensures that the fluorescence is evenly dispersed throughout the scaffold. All further work with the fluorescent PLGA was performed in the dark.

Fabrication of 3D scaffolds Fluorescently labelled PLGA film was dissolved in chloroform (10\% w/v) with $50 \%$ sodium chloride (Sigma-Aldrich, UK) with particle diameter size of $100-150 \mu \mathrm{m}$. This solution was placed in a 2 $\mathrm{mL}$ syringe and lypholised overnight at $-50{ }^{\circ} \mathrm{C}$ under a vacuum to evaporate the solvent. The scaffold was then removed from the syringe and cut into discs with a $1 \mathrm{~mm}$ thickness. The discs were then placed in distilled water for 2 hours under stirrer conditions at $200 \mathrm{rpm}$ to remove the sodium chloride (Sigma-Aldrich, UK) within the scaffold. The scaffolds were once again lypholised overnight at $-50^{\circ} \mathrm{C}$ under a vacuum.

\section{Characterisation of Fluorescent PLGA}

X-ray Photo Spectrometry (XPS) XPS results were obtained by the NEXUS laboratory (Newcastle, UK), using the Thermo K-Alpha XPS instrument (Thermo Scientific, East Grinstead, UK). A microfocused monochromatic alK $\alpha$ Xray source with energy of $1486.6 \mathrm{eV}$, voltage $12 \mathrm{kV}$, current of $3 \mathrm{~mA}$ and power $36 \mathrm{~W}$ and a $400 \mu \mathrm{m}$ spot size was utilized for the analysis. 50 survey scans were performed at $200 \mathrm{eV}$ with a dwell time of $10 \mathrm{~ms}$. High resolution regions were scanned 10 times at $40 \mathrm{eV}$ with a dwell time of $50 \mathrm{~ms}$. XPS was performed on PLGA films at three stages of labelling: un-labelled PLGA, ammonia plasma coated PLGA, ammonia and rhodamine $b$ isothiocyanate labelled PLGA. A control sample of rhodamine modified PLGA without ammonia plasma treated was also scanned to investigate non-specific binding.

High Performance Liquid Chromatography (HPLC) Fluorescently labeled PLGA films were completely dissolved in a mixture solvent of $80 \%$ acetonitrile in distilled water with $0.1 \%$ trifluoroacetic acid (TFA) (Fisher Scientific, UK) for 2 days $(10 \mathrm{mg} / \mathrm{mL})$, the solution was then filtered and run through a reverse phase 1220 LC system VL (Aglient) using a Jupiter $5 \mathrm{u}$ column (Phenomenex). The molecules exiting the column were monitored for 30 mintues via a UV detector at $550 \mathrm{~nm}$, the absorbance of rhodamine $b$ isothiocyanate. The retention peaks were then compared to a rhodamine standard ( $70 \mu \mathrm{g} / \mathrm{mL}$ in $80 \%$ acetonitrile with $0.1 \% \mathrm{TFA})$.

Gel Permeation Chromatography (GPC) PLGA molecular weight was analysed using a Thermo Ultimate 3000 UHPLc with a mobile chloroform phase $\left(1.0 \mathrm{~mL} /\right.$ minute), at $40{ }^{\circ} \mathrm{C}$. The GPC consisted of two PLgel Mixed C columns $(300 \times 7.5 \mathrm{~mm}$ ) with a $5 \mu \mathrm{m}$ particle size (Agilent, USA) connected in series and protected by a PLgel Mixed C guard column. Calibration was performed using Agilent EasiCal polystyrene standards (Agilent, USA). PLGA samples were prepared in chloroform, $25 \mathrm{mg} / \mathrm{mL}$, for analysis and $50 \mu \mathrm{L}$ was injected per run.

Hydrophobicity Contact angle was used to assess the hydrophobicity of the PLGA films. A $0.2 \mathrm{~mL}$ droplet of distilled water was placed on the film surface and the equilibrium contact angle measured.

\section{Assessment of PLGA Degradation}

The degradation of porous 3D PLGA scaffolds ( $8 \mathrm{~mm}$ diameter $x 1 \mathrm{~mm}$ thickness) was quantified by the change in physical weight, fluorescence retention and the release of soluble fluorescent by-products into the degradation solution, phosphate buffered saline (PBS) (SigmaAldrich, UK) during the incubation of the scaffolds at $37^{\circ} \mathrm{C}$ in a humidified atmosphere. Control non-modified scaffolds were assessed by changes in physical weight alone.

To assess changes in the physical weight of the scaffold the PLGA was removed from the PBS solution, and placed on blotting paper for 30 seconds, to remove excess water from the surface, before weighing. To investigate the release of fluorescence into the PBS a $200 \mu \mathrm{L}$ sample was taken and the fluorescence intensity (Excitation: 530/ Emission: 590) was measured using a fluorescence 
plate reader (BioTek Synergy II). This was then compared to calibration curve of known concentrations of fluorescent PLGA.

Confocal laser scanning microscopy (CLSM) (Olympus, UK) was utilised to assess the fluorescence intensity retained within the PLGA throughout the degradation period. 3D visualisation of the PLGA was achieved by scanning through the scaffold at $10 \mu \mathrm{m}$ intervals over a range of $200 \mu \mathrm{m}$ from the base of the scaffold (z-axis) with a $\times 10$ long working distance, air lens (Olympus, UK). The imaging parameters were maintained throughout the degradation period and changes in the average peak fluorescence values were calculated and normalized to a standard undegraded scaffold at each time point to account for internal variations within the system.

\section{Ex Vivo Chick Femur Model}

Embryonic femurs were dissected from Dekalb White chicks (MedEggs, UK) at E11 and excess tissue excluding muscle and tendon was carefully removed. The femurs were cultured organotypically on $0.4 \mu \mathrm{m}$ filter well inserts within a 6 well with $1 \mathrm{~mL}$ of osteogenic media (low glucose $(1 \mathrm{~g} / \mathrm{L}$ ) Dulbecco's Modified Eagle Media (DMEM) (Lonza, UK), $2 \mathrm{nM}$ L-glutamine (Lonza, UK), $25 \mathrm{U}$ Penicillin/25 U Streptomycin (Lonza, UK), $10 \%$ fetal calf serum (Biosera, UK), $10 \mathrm{mM} \beta$-glycerophosphate (Sigma-Aldrich, UK), $100 \mathrm{nM}$ dexamethasone (Sigma-Aldrich, UK) and $50 \mathrm{\mu g} / \mathrm{mL}$ ascorbic acid (sigma-Aldrich, UK)) at $37^{\circ} \mathrm{C}$ in humidified air with $5 \% \mathrm{CO} 2 .{ }^{16} 24$ hours after isolation a 0.5 $\mathrm{mm}$ drill defect was formed in the epiphyseal end of the femur and a porous fluorescent PLGA plug was implanted within the defect. The degradation of the various PLGA compositions (50/50, 65/35 and 80/20) was then monitored using an in vivo imaging system (PhotonImager RT, Biospace Lab, France).

\section{Results and Discussion}

The use of biomaterials within the field of tissue engineering is widespread with the rate of degradation being an essential property of these materials. The majority of current research relies on end point assays for the assessment of biomaterial degradation, especially when investigating polymers such as PLGA which have no characterized molecular marker in degradation products. In this paper we have described and characterised a fluorescent PLGA, which will allow for the online, real-time monitoring of degradation in a non-destructive manner.

After PLGA surface modification through ammonia plasma treatment the rhodamine $b$ isothiocyanate was shown to be taken up by the PLGA films and was retained throughout post reaction washing as shown by CLSM. It was observed that fluorescence was present on the modified surfaces of the PLGA films, with the highest peak being observed on the top surface of the film during treatment (Figure 1a). Importantly once the modified films were recast into new films or 3D saltleached scaffolds the fluorescence was observed to be evenly distributed throughout the PLGA (Figure 1a), which is essential for investigating the degradation profile.

It was observed that that areas of PLGA covered during the plasma treatment were not fluorescently modified by the rhodamine $b$ isothiocyanate (Figure $1 b$ ), this suggests that the specificity of the reaction is limited to areas which are modified with reactive amine groups. Uptake rates, before washing, (Figure 1c) and retention rates, after washing, (Figure 1d) of rhodamine b isothiocyanate in PLGA films, however were shown to correlate to the hydrophilicity of the PLGA compositions. It was observed that the more hydrophilic films (50:50 and 65:35), with a higher swelling degree, were shown to initially take up more rhodamine $b$ isothiocyanate before washing (Figure 1c). Therefore due to the innate swelling properties of the PLGA it was essential to ensure that the rhodamine was covalently bound to the modified PLGA and not merely entrapped during the natural material swelling. In order to fully represent degradation it is essential that the rhodamine $b$ isothiocyanate is bound to the PLGA and that random leaching of the fluorescence out of the scaffold does not occur as this will affect the accuracy of the degradation calibration.

XPS survey scans of the plasma treated PLGA showed a new ammonia peak in the spectra (Figure 2a) on the surface, this is confirmed when looking at the both high resolution ammonia and carbon scans (Figure $2 \mathrm{~b}$ and Figure $2 \mathrm{c}$ respectively). High resolution ammonia scans showed peaks in both the plasma treated, and plasma treated, rhodamine $b$ isothiocyanate 
grafted PLGA (Figure 2b). High resolution carbon spectra showed a shift in the carbon-carbon/carbon-hydrogen (C-C/C-H) binding energy due to the formation of higher energy carbon-nitrogen (C-N) bonds as the PLGA surface was modified (Figure 2c). The change in the surface chemistry of the PLGA films confirms the appearance of isothiocyanate reactive amine groups. Plasma treatment has been previously been used to modify PLGA surfaces in order to affect the cellmaterial interactions. For example oxygen and ammonia plasma treatment have both been shown to improve cell attachment and cell proliferation. ${ }^{17,18}$ The positive effect of amine modified surfaces suggests that any un-reacted amine groups left within the PLGA will not have a detrimental effect on cell attachment or proliferation and may increase cellmaterial interactions.

After rhodamine $\mathrm{b}$ isothiocyanate was reacted with the amine modified PLGA an increased peak in C-C/C-H bonds was observed due to the high number of these bonds within the rhodamine $b$ isothiocyanate. This was confirmed through HPLC of degraded PLGA, which showed variation in the polarity of fluorescent products when compared to the rhodamine standard (Figure $3 a$ and Figure $3 b$ respectively). This increase in the number and the retention time of the peaks when compared to the rhodamine standard indicated the formation of highly polar fluorescent monomers and dimers of lactic/glycolic acid. However, a slight increase in the peak intensity for the $\mathrm{C}-\mathrm{C} / \mathrm{C}-\mathrm{H}$ bonds was also observed in the nonammonia plasma treated, rhodamine treated samples indicating that there was some non-specific uptake of the rhodamine. When investigating the XPS spectra no peak changes are observed in the hydroxyl (C-OH) or the carbonyl groups $(C=O)$ showing there was no non-specific binding of rhodamine $b$ isothiocyanate in the absence of the ammonia plasma coating. This suggests that some of the rhodamine may remain unbound within the film during the modification of the PLGA, due to the swelling.

It was essential to also ensure that the modification of the PLGA did not turn cause alterations in polymer chemistry, which may in turn affect the degradation rate. Molecular weight and hydrophobicity of the PLGA are two such factors which could have been affected by polymer modification. It was shown that the neither the molecular weight nor hydrophobicity were significantly altered through the fluorescent modification (Table 1). Both of these parameters have been shown to affect the degradation of PLGA, with decreased molecular weight and increased hydrophilicity increasing hydrolysis of the polymer. ${ }^{19}$ Therefore the fact that these parameters are not altered is essential for accurate monitoring of PLGA degradation.

After fluorescent labelling the PLGA was re-cast in order to disperse the fluorescence evenly across the depth of PLGA films (Figure 1a) and across the transverse section of the three-dimensional scaffolds (Figure 4) allowing for accurate determination of the degradation profile. It was however essential to determine the labelling efficiency and therefore the fluorescent signal per mg of PLGA. It was shown that the labelling efficiency was varied dependent on the PLGA composition; however there was a high correlation between the weight and fluorescence, with Pearson's $r$ values of $0.9842,0.9549$ and 0.9589 for 50/50, 65/35 and 80/20 PLGA composition respectively. (Figure 5)

During degradation the fluorescence retained within the PLGA scaffolds was depleted, for all compositions, when monitored by CSLM (Figure 4a and Figure 4b). This depletion was shown to vary depending on the PLGA composition with higher glycolic acid content leading to increased fluorescence reduction when compared to the slower degrading PLGA compositions with higher lactic acid content (Figure 4b). This change in fluorescence concurred with fluorescence release into the media, correlated to weight using calibration curves (Figure 5) and changes in the physical wet weight of the polymer for all three of the polymer

compositions 50/50, 65/35 and 80/20 (Figure 6a, Figure $6 \mathrm{~b}$ and Figure $6 \mathrm{c}$ respectively). Overall, the measurements demonstrate an excellent correlation between weight loss, fluorescence retention and the release of soluble fluorescent by-products, with the all biomaterials exhibiting comparable weight losses independent of the parameters measured (Figure 6). This novel method for labelling inert PLGA with a fluorescent tag, therefore enables accurate monitoring of the degradation of the scaffolds, in vitro. This is essential as it allows for online monitoring of biomaterial degradation of the same sample over a period of time. It has previously been reported that the degradation of fluorescent biomaterials can be monitored intra-vitally both in vitro and in vivo, decreasing the required number of samples and allowing for continual monitoring of the same specimen. ${ }^{7}$ PLGA mainly degrades through hydrolysis and therefore the degradation profiles in vitro and in vivo should correlate. However PLGA degradation has also been shown to vary with dimension and environmental conditions, such as $\mathrm{pH}$ and mechanical stresses and strains, which may be present in vivo that are not present in vitro and vice versa. ${ }^{20-22}$ It has also been suggested that, in vivo, enzymes may play a role in the degradation of PLGA, although there has been difficulty in identifying enzymes and their contributions. ${ }^{22}$

Degradation profiles of the various PLGA compositions were, therefore, also observed within the epiphyseal defect of the organotypic ex vivo chick femur and the percentage degradation quantified by the change in average photons emitted across the defect (Figure 7). The degradation of the PLGA 50/50 was once again shown to be the fastest degrading polymer 
as assessed by changes in fluorescence. The degradation profile of the PLGA compositions was however altered from that seen in vitro (Figure 6), with slower degradation observed within the ex vivo chick femur model (Figure 7). These variations confirm that it is essential for biomaterial degradation to be investigated both in vitro and in vivo, in order to ensure that the profile is not altered to such an extent that will impact on tissue regeneration.

The change observed in the degradation profile from in vitro to ex vivo may also be due to the fact that the fluorescent degradation products may not be able to diffuse through the tissue and disperse. This method of fluorescent tagging therefore may also allow for the tracking of degradation by-products of the biomaterials used in tissue engineering. This is particularly important when investigating biomaterials where the accumulation of by-products may have a deleterious effect on the tissue repair. The degradation of PLGA in particular is known to produce an acidic local environment, because of the glycolic and lactic acid monomer by-products. In turn low pH environments are shown to have deleterious effects on proteins and nucleic acid. ${ }^{23}$ Therefore the ability to track fluorescent by-product diffusion and accumulation throughout tissues, may allow for better treatment design and maybe essential for the regulatory approval of these scaffold materials.

When investigating in vivo degradation it is also essential that the chosen fluorochromes or tag is able to penetrate the required tissue depth. Due to the high auto-fluorescence of both skin and fur, it is essential that the correct animal model and fluorochrome is selected. Fluorochomes that emit toward the red end of the spectra are of particular interest, as water and hemoglobin absorb less of the photons, therefore allowing for deeper tissue penetration. ${ }^{24}$ This novel method of tagging PLGA through the formation of a reactive amine surface has the advantage of being able to be used on a variety of polymers, which have no reactive groups intrinsically, and with a range of amine reactive tags. There are several different fluorescent amine reactive moieties which could be used to bind a range of fluorochromes, such as fluorescein and rhodamine, these include isothiocyanate groups, used in this research, succinimidyl ester groups and dichlorotriazine groups. ${ }^{25}$ This ability to interchange between fluorochromes allows for the monitoring of multiple degradation profiles, which opens up the ability to investigate material interactions. For example polyglycolic acid and poly-L-lactic acid could be individually monitored to determine the individual degradation profiles of the two materials. This method could also be utilized for other methods of imaging, such as photoacustic imaging using other amine reactive compounds such as, Monocarboxymethylene Blue $\mathrm{N}-\mathrm{Hydroxysuccinimide} \mathrm{ester.}{ }^{26}$

\section{Conclusions}

This study has described a novel fluorescent tagging method for PLGA to monitor the degradation profile of the biomaterial in a non-destructive manner. This novel method of using plasma treatment and amine reactive fluorochromes offers the possibility to tag other inert polymers with a wide range of fluorochromes, to investigate real-time degradation. This ability to monitor biomaterial degradation both in vitro and in vivo is essential not only within the 3Rs policy but also for regulatory approval as it allows for the tracking of not only biomaterial degradation but also by-product accumulation or diffusion.

\section{Acknowledgements}

The authors would like to thank the EU-FP7 BioDesign 'Rational Bioactive Materials Design for Tissue Regeneration' Project for funding (\#262948). X-ray photoelectron spectra were obtained at the National EPSRC XPS Users' Service (NEXUS) at Newcastle University, an EPSRC Mid-Range Facility. We would also like to thank Locate Therapeutics Limited, Nottingham, UK, for running GPC analysis and Dr Wenwu Li for HPLC support.

\section{References}

1. M.N. Rahaman, D.E. Day, B.S. Bal, Q. Fu, S.B. Jung, L.F. Bonewald and A.P. Tomsia, Acta Biomaterialia, 2011, 7, 2355.

2. R. Langer and J.P. Vacanti, Science, 1993, 260, 920.

3. M. Nagata, A. Oi, W. Sakai and N. Tsutsumi, Journal of Applied Polymer Science, 2012, 126, E358.

4. P. Giunchedi, B. Conti, S, Scalia and U. Conte, J Control Release, 1998, 56, 53.

5. C.S. Proikakis, N.J. Mamouzelos, P.A. Tarantili and A.G. Andreopoulos, Polymer Degradation and Stability, 2006, 91, 614 .

6. G.H. van Lenthe, H. Hagenmüller, M. Bohner, S.J. Hollister, L. Meinel and R. Müller, Biomaterials, 2007, 28, 2479.

7. N. Artzi, N. Oliva, C. Puron, S. Shitreet, S. Artzi, A. bon Ramos, A. Groothuis, G. Sahagian and E.R. Edelman, Nat Materials, 2011, 10, 704. 
8. C. Cunha-Reis, A.J. El Haj, X. Yang and Y. Yang, Journal of Tissue Engineering and Regenerative Medicine, 2013, 7, 39.

9. S. Wolbank, V. Pichler, J.C. Ferguson, A. Meinl, M. van Griensven, A. Goppelt and H. Redl, Journal of Tissue Engineering and Regenerative Medicine, 2015, 9, 973.

10. N.J. Osborne, D. Payne and M.L. Newman, The American Journal of Bioethics, 2009, 9, 55.

11. H. Ueda and Y. Tabata, Advanced Drug Delivery Reviews, 2003, 55, 501.

12. J.M. Karp, M.S. Shoichet and J.E. Davies, Journal of Biomedical Materials Research Part A, 2003, 64A, 388.

13. Y. Zhang, F. Yang, K. Liu, H. Shen, Y. Zhu, W. Zhang, W. Liu, S. Wang, Y. Cao and G. Zhou, Biomaterials, 2012, 33, 2926.

14. K.J. Aviss, J.E. Gough and S. Downes, Eur Cell Mater, 2010, 19, 193.

15. Z. Karahaliloğlu, B. Ercan, S. Chung, E. Taylor, E.B. Denkbas and T.J. Websteet, Journal of Biomedical Materials Research Part A, 2014, 102, 4598.

16. J.M. Kanczler, E.L. Smith, C.A. Roberts and R.O.C Oreffo, Tissue Eng Part C Methods, 2012, $18,747$.

17. K. Kato, E. Uchida, E. Kang, Y. Uyama and Y. Ikada, Progress in Polymer Science, 2003, 28, 209.

18. T. Jacobs, R. Morent, N. De Geyter, P. Dubruel and C. Leyset, Plasma Chemistry and Plasma Processing, 2012, 32,1039.

19. H. Keles, A. Naylor, F. Clegg and C. Sammon, Polymer Degradation and Stability, 2015, 119, 228.

20. Grizzi, H. Garreau, S. Li and M. Vertet, Biomaterials, 1995, 16, 305.

21. B.S. Zolnik and D.J. Burgess, Journal of Controlled Release, 2007, 122, 338.

22. H.K Makadia, and S.J. Siegel, Polymers, 2011, 3, 1377.

23. K. Fu, D.W. Pack, A.M. Klibanov, and R. Langer, Pharmaceutical Research, 2000, 17, 100.

24. V. Ntziachristos, C. Bremer and R. Weissleder, European Radiology, 2003, 13, 195.

25. P.R. Banks and D.M. Paquette, Bioconjugate Chemistry, 1995, 6, 447.

D. Wu, L. Huang, M.S. Jiang and H. Jiang, International Journal of Molecular Sciences, 2014, 15, 23616 


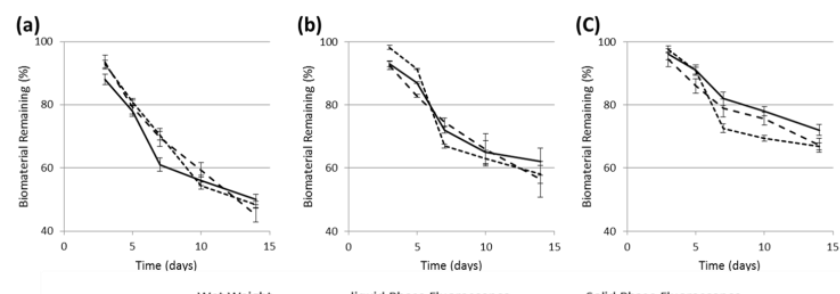

Figure 6. Correlation of biomaterial degradation, (a) PLGA 50/50, (b) PLGA 65/35 and (c) PLGA 80/20. Degradation was monitored over a 14 day period in PBS using changes in wet weight, retention of fluorescence within the solid phase of the biomaterials and the release of soluble fluorescent by-products into the liquid phase. The three methodologies for monitoring degradation showed similar degradation trends across all PLGA compositions. Error bars are drawn as standard error of the mean, $n=6$

(a)
\begin{tabular}{|c|c|}
\hline PLGA Composition & Labelling Efficiency (\%) \\
\hline $50 / 50$ & 11.97 \\
\hline $65 / 35$ & 9.54 \\
\hline $80 / 20$ & 20.43 \\
\hline
\end{tabular}
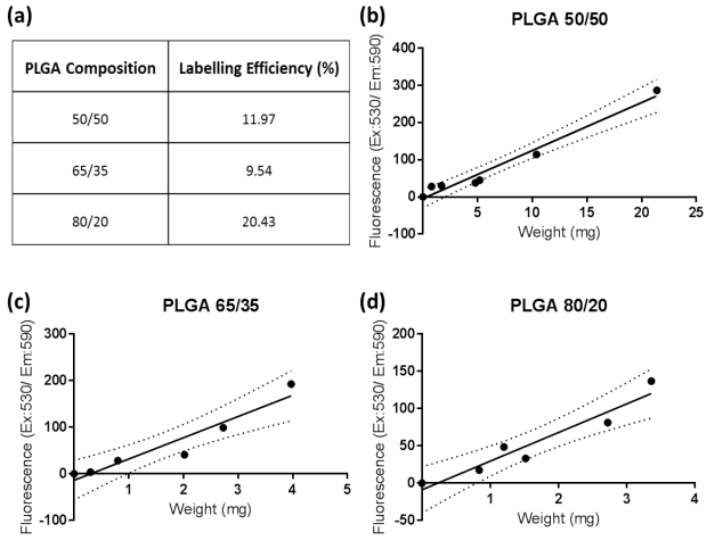

Figure 5. (a) Molar labelling efficiency of the varying PLGA compositions, (b)-(d) calibration curve showing weight (mg) to fluorescence emission (Ex:530/Em:590) for varying PLGA compositions; (b) 50/50, (c) 65/35 and (d) 80/20.
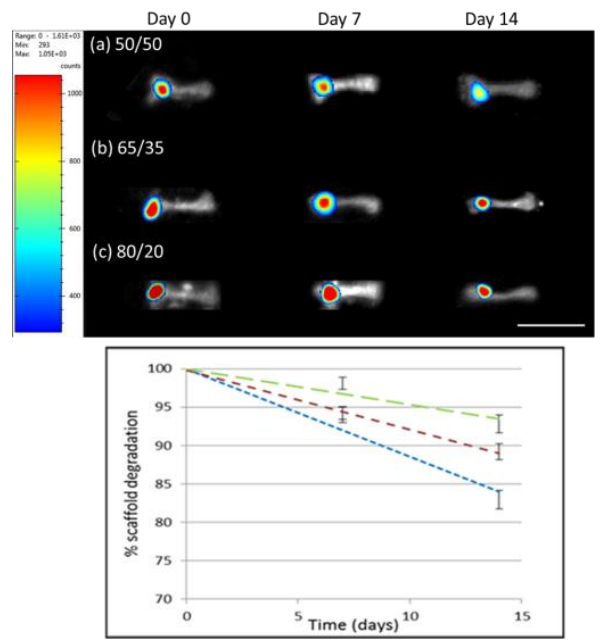

Figure 7. PLGA degradation profiles in an ex vivo chick femur model. (a-c) the visualised degradation of PLGA 50/50, 65/35 and $80 / 20$ respectively, showing a decrease in fluorescence intensity with time (high photon count - red, low photon count blue). (d) Quantification of fluorescence degradation through the calculation of average photon counts across the fluorescent PLGA. 


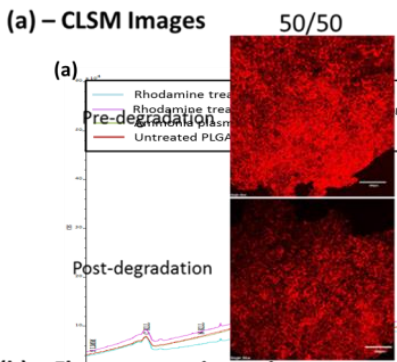

(b) - Fluorescence intensity
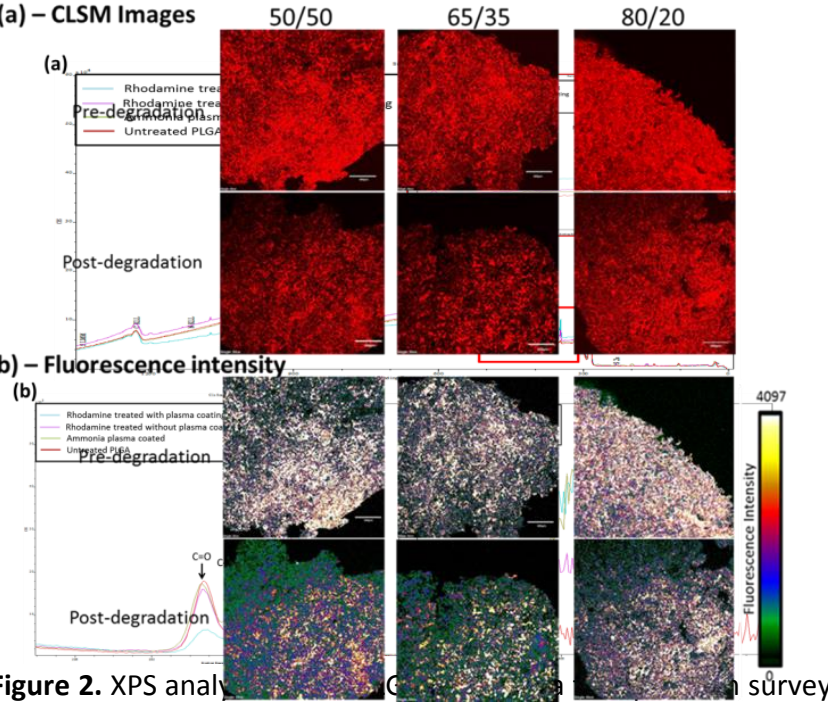

showing the appearance of nitrogen peaks on the plasma coated scaffolds (blue and green), (b) high resolution carbon spectra displaying a left shift in carbon-carbon and carbon-hydrogen bond peak caused by the appearance of higher energy carbon-nitrogen bonds and (c) high resolution nitrogen spectra showing the appearance of nitrogen peaks in the ammonia plasma treated PLGA films.

(a)

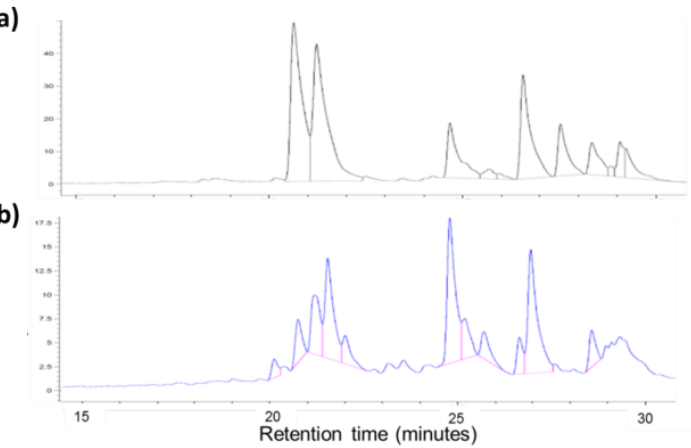

Figure 3. HPLC analysis of PLGA modified films dissolved in $80 \%$ acetonitrile with $0.1 \%$ TFA showing the differences in peak retention times of $(\mathrm{a})$ rhodamine control and $(\mathrm{b})$ the rhodamine b isothiocyanate modified PLGA.

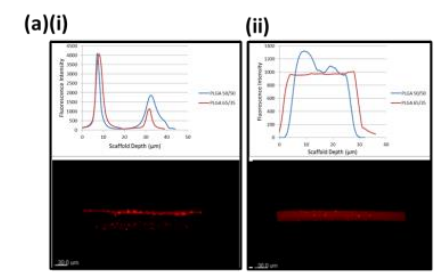

(c)
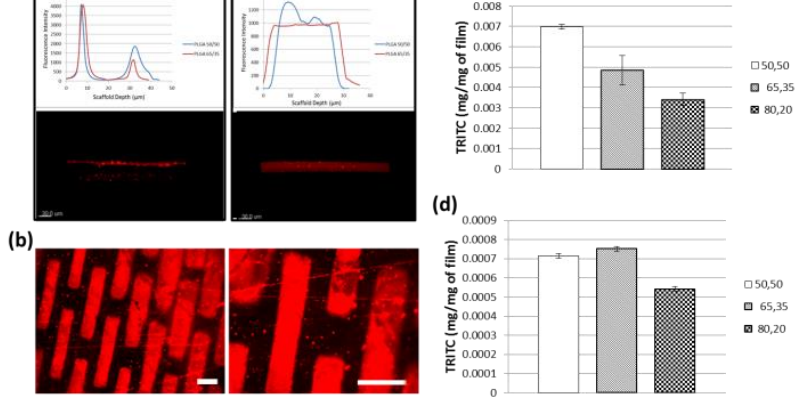

Figure 1. Initial analysis of rhodamine $b$ isothiocyanate modified films showing (a) the appearance of red fluorescence on the surface of the films prior to recasting (a)(i) and evenly distributed throughout the depth of the PLGA after recasting (a(ii)), as deciphered by CLSM intensity plots and images. (b) Masked areas of the PLGA film during plasma coating did not take up fluorescence after treatment with rhodamine b isothiocyanate. (c) The uptake of rhodamine $b$ isothiocyanate before washing showing the increase in more hydrophilic films. (d) The retained rhodamine $b$ isothiocyanate on the PLGA films after extensive washing with distilled water and $70 \%$ ethanol.

Figure 4. Fluorescence $(A)$ and intensity (B) images of the porous PLGA scaffolds as obtained by CLSM, showing the effect of degradation on the fluorescence in the $x / y$ plane on the various PLGA compositions after the 14 day degradation period. Fluorescence was measured using a wavelength of $561 \mathrm{~nm}$. 